\title{
Response to: Comment on "Prevalence, Etiology, and Risk Factors of Tinea Pedis and Tinea Unguium in Tunisia"
}

\author{
Nourchène Toukabri $\mathbb{D}^{1},{ }^{1}$ Cyrine Dhieb, ${ }^{1}$ Dalenda El Euch, ${ }^{2}$ Mustapha Rouissi, ${ }^{3}$ \\ Mourad Mokni, ${ }^{2}$ and Najla Sadfi-Zouaoui $\mathbb{D}^{1}$ \\ ${ }^{1}$ Laboratoire de Mycologie, Pathologies et Biomarqueurs, Faculté des Sciences de Tunis, Université Tunis El Manar, \\ 2092 Tunis, Tunisia \\ ${ }^{2}$ Service de Dermatologie et de Vénéréologie, Hôpital La Rabta, Tunis, Tunisia \\ ${ }^{3}$ Institut National de la Recherche Agronomique de Tunis, Tunis, Tunisia
}

Correspondence should be addressed to Najla Sadfi-Zouaoui; sadfi.najla@gmail.com

Received 20 March 2018; Accepted 28 March 2018; Published 22 May 2018

Academic Editor: Maria L. Tornesello

Copyright (C) 2018 Nourchène Toukabri et al. This is an open access article distributed under the Creative Commons Attribution License, which permits unrestricted use, distribution, and reproduction in any medium, provided the original work is properly cited.

We are grateful to Dr. Talel Badri [1] for his comments and suggestions on the article "Prevalence, Etiology, and Risk Factors of Tinea Pedis and Tinea Unguium in Tunisia" [2].

We have carefully looked at all the comments and earnestly hope that the provided responses will fulfill the concerns.

The statistical analysis of predisposing factors of foot mycosis using odds ratio and multivariate analysis "PCAMCA" is explained in the following paragraph in accordance with Table 1.

Statistical analysis was performed with SPSS software (Statistical Package for Social Sciences version 20.0, SPSS Inc., Armonk, NY). Odds ratio (OR) with $95 \%$ confidence interval (CI) was measured. The chi-square $\left(\chi^{2}\right)$ was also used to calculate significant differences in characteristics between patients. Differences with $p<0.05$ were considered statistically significant. Multivariate analyses were carried out by two methods: principal components analysis (PCA) and multivariate correspondence analysis (MCA).

Table 1 shows results of odds ratio, 95\% CI, and $P$ value; as a conclusion from Table 1, factors associated with foot mycosis such as sex, nail trauma, peripheral vascular disease, psoriasis, and age group over 51 do not present risk factors, whereas presence of dermatological pathology, obesity, wearing used shoes, occlusive shoes, smoking, attending swimming pools, making pedicure, and presence of fungal infection of the skin represent a risk factor of foot mycosis. However, practicing ritual washing, physical activities, attending communal shower, presence of family history, trauma of the nail, application of henna, walking barefoot, using thermal station, presence of associated fingernails, diabetes, and immunosuppressive therapy are protective factors.

Consequently, subjects especially between the age group 40 and 50 seem to be the most exposed to foot mycosis.

Multivariate analysis was carried out to determine the relationship between factors associated with foot mycosis. The correlation between factors, as shown in Table 2, discriminates factors highly related from those having a weak relationship with the infection.

For example, obesity is highly correlated to diabetes and peripheral vascular disease; however, wearing occlusive shoes is not correlated to ritual washing and attending communal shower.

As a result from this correlation matrix and the PCA (principal components analysis), factors are distributed as shown in Figure 1. From this illustration, we conclude that we have three groups of factors: most factors positively close to PC2 axis seem to be risk factors for foot mycosis, whereas most factors positively close to PC1 axis (sex, family history, wearing used shoes, nail trauma, immunosuppressive drugs, season, and peripheral vascular disease) seem to be with no 


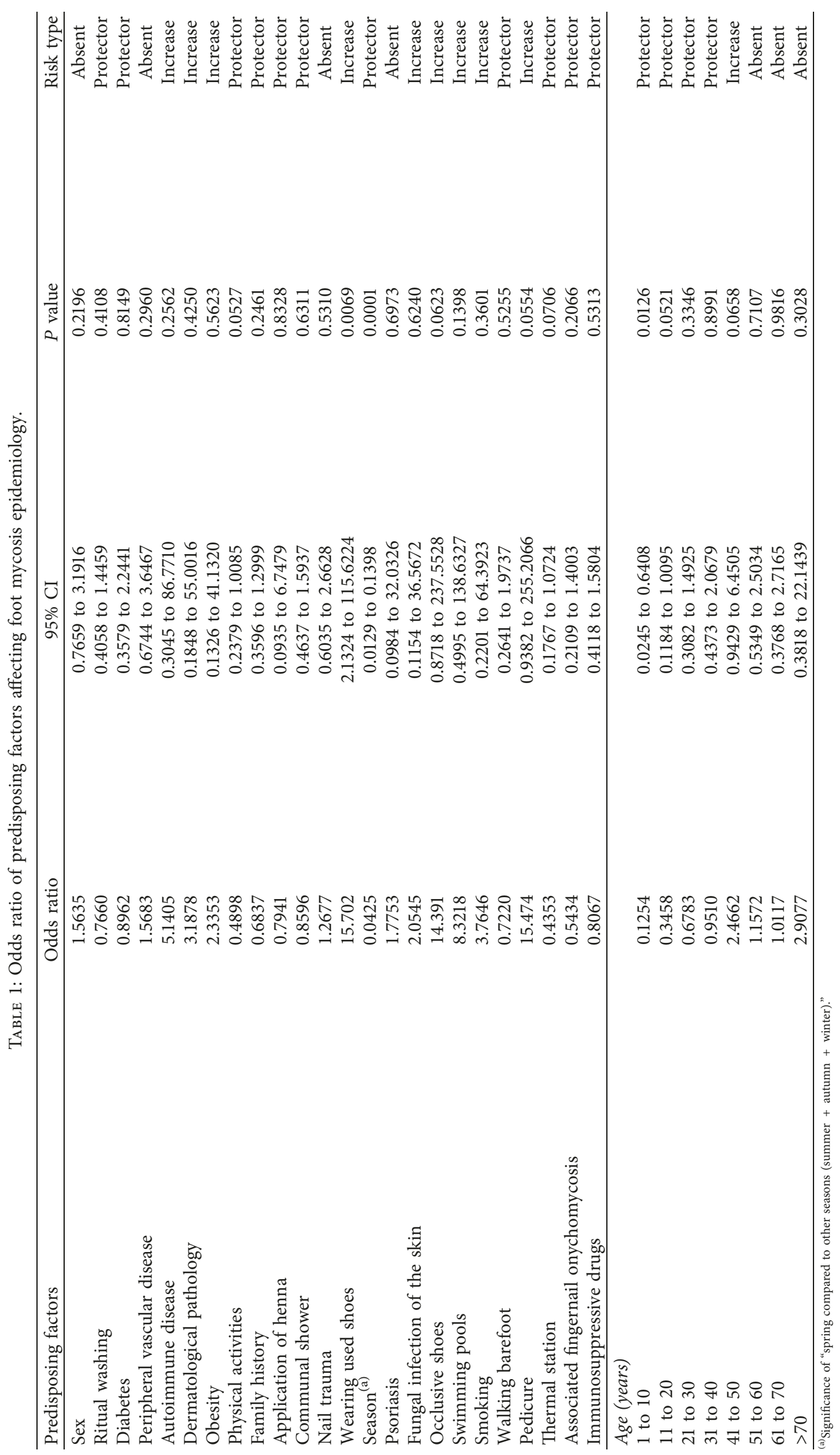




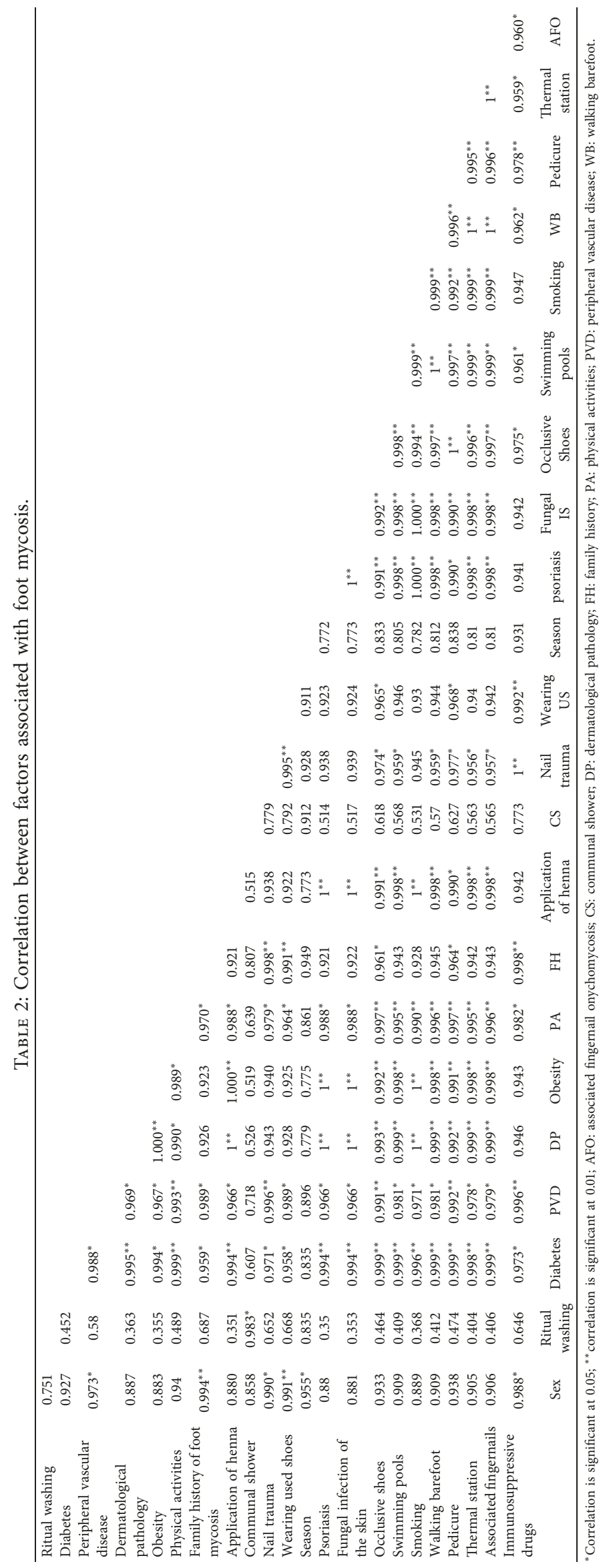




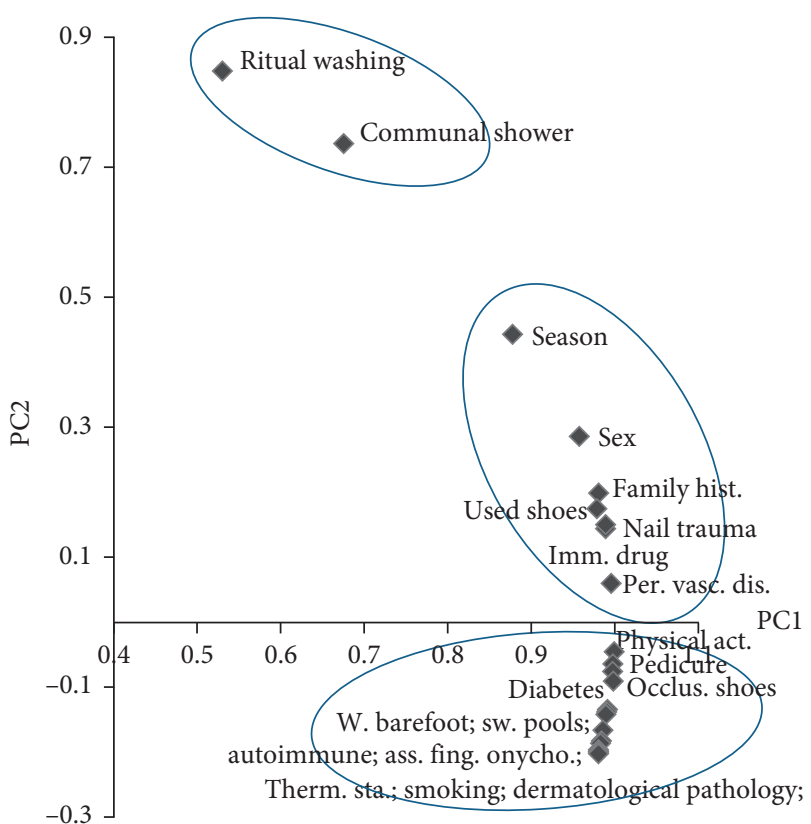

FIGURE 1: Principal components diagram of factors associated with foot mycosis.

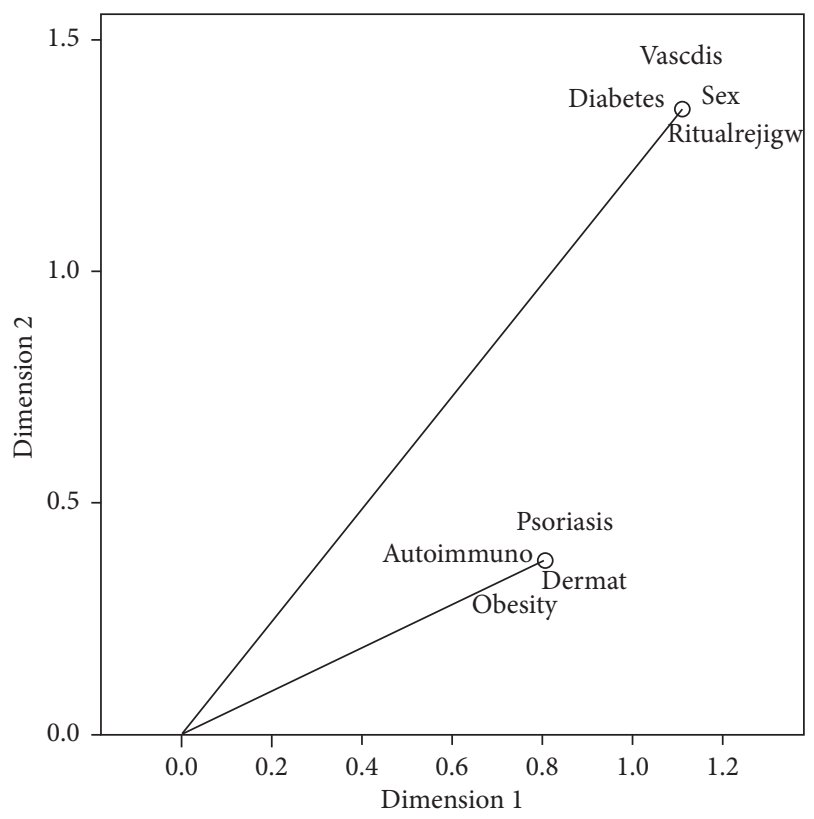

FIGURE 2: Multiple correspondence analysis of factors associated with foot mycosis.

risk; however, the group of factors negatively close to PC1 axis (physical activities, pedicure, occlusive shoes, diabetes, walking barefoot, swimming pools, smoking, thermal station, associated fingernail onychomycosis, dermatological pathology, obesity, fungal infection of the skin, psoriasis, application of henna, and autoimmune disease) are protectors from risk.

We have also carried out a second multivariate analysis which is MCA (multiple correspondence analysis) in order to compare results of the two multivariate methods.
A plot illustrating eignvectors of factors associated with foot mycosis allows to discriminate between two essential groups: the first group gathering the sex, peripheral vascular disease, diabetes, and ritual washing, and the second including psoriasis, autoimmune disease, dermatological pathology, and obesity (Figure 2).

From this work and depending on odds ratio analysis and the two multivariate methods used, we can conclude that we generally obtain the same groups of factors with some difference depending on the performance of methods.

It is true that there is a selection variation in our series. The clinical consultants are much more prone to suggest the mycological test in tinea unguium than tinea pedis because of the duration and the cost of treatment.

In order to respond to the last comment, we have a certitude explanation of nonsignificance of the two factors (ritual washing and communal shower), but it may be related to the improvement of hygiene in these facilities (mosques and hammams) and the individual behavior.

\section{Conflicts of Interest}

The authors declare that they have no conflicts of interest.

\section{References}

[1] T. Badri, "Comment on "prevalence, etiology, and risk factors of tinea pedis and tinea unguium in Tunisia", Canadian Journal of Infectious Diseases and Medical Microbiology, vol. 2018, Article ID 4859514, 1 page, 2018.

[2] N. Toukabri, C. Dhieb, D. El Euch, M. Rouissi, M. Mokni, and N. Sadfi-Zouaoui, "Prevalence, etiology, and risk factors of tinea pedis and tinea unguium in Tunisia," Canadian Journal of Infectious Diseases and Medical Microbiology, vol. 2017, Article ID 6835725, 9 pages, 2017. 


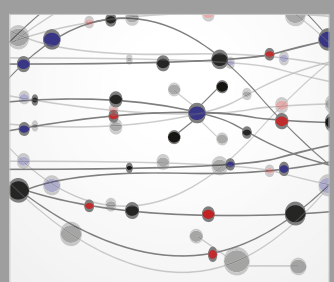

The Scientific World Journal
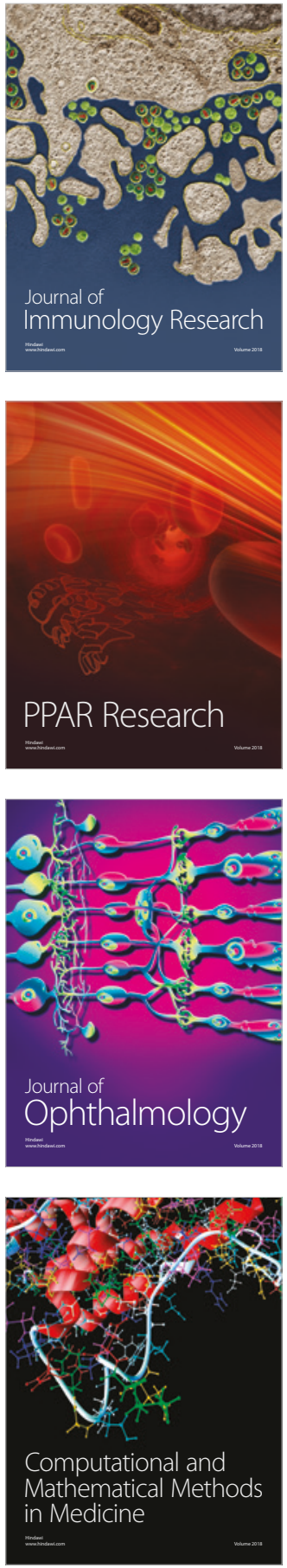

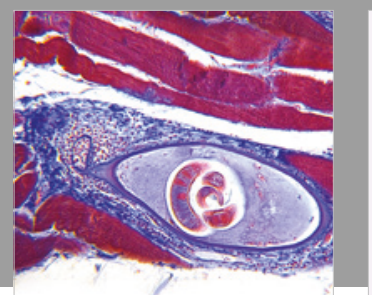

Gastroenterology Research and Practice

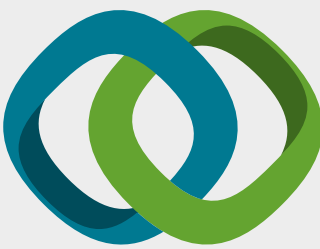

\section{Hindawi}

Submit your manuscripts at

www.hindawi.com
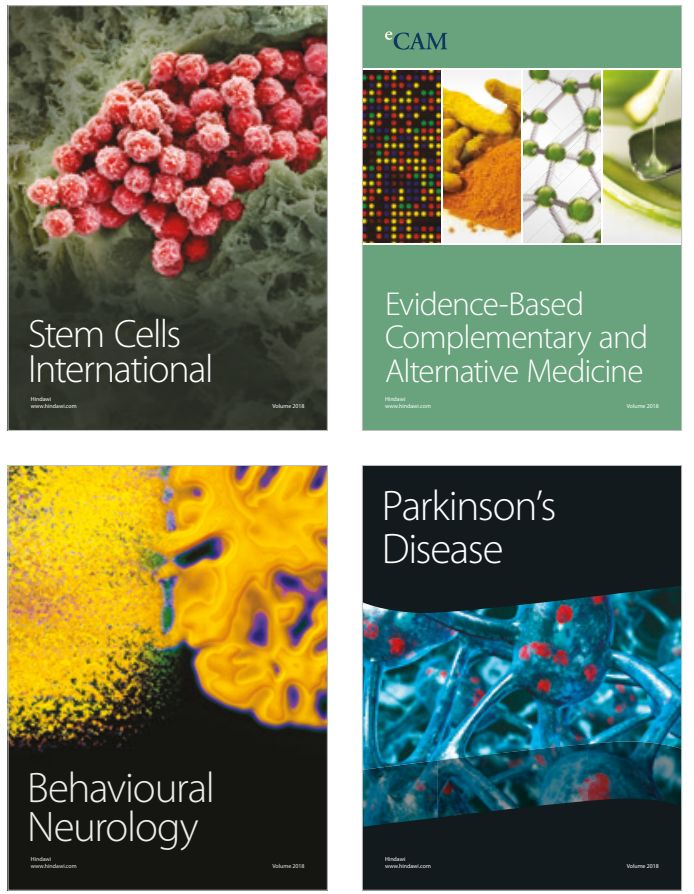

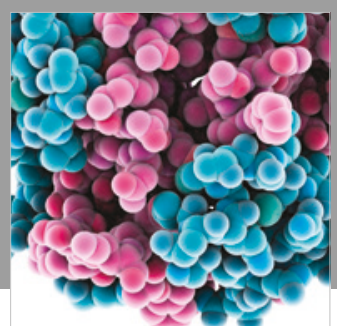

ournal of

Diabetes Research

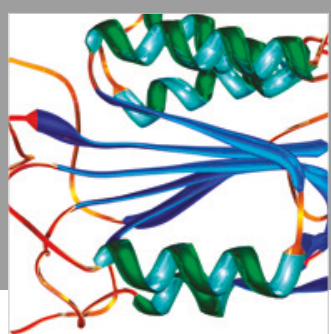

Disease Markers
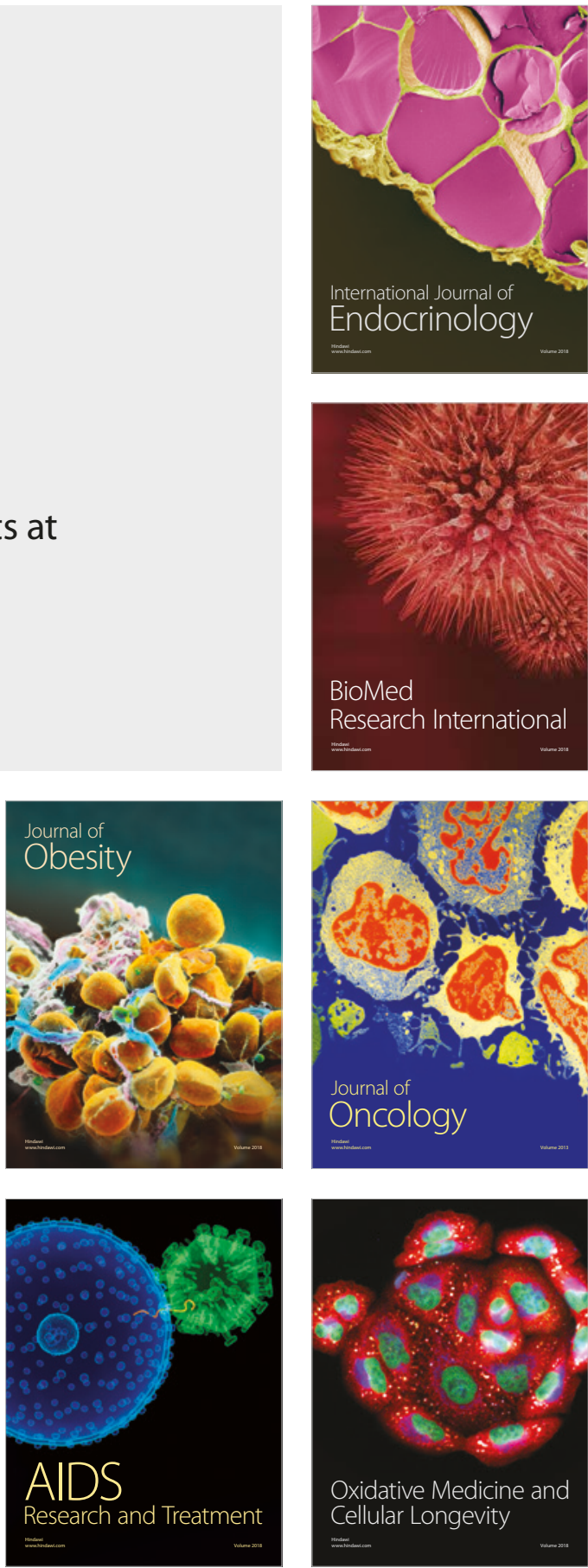